September 3, 2018

\title{
Qubit measurement by a quantum point contact: a quantum Langevin equation approach
}

\author{
Bing Dong,,$^{1,2}$ Norman J. M. Horing, ${ }^{2}$ and X. L. Lei ${ }^{1}$ \\ ${ }^{1}$ Department of Physics, Shanghai Jiaotong University, 1954 Huashan Road, Shanghai 200030, China \\ ${ }^{2}$ Department of Physics and Engineering Physics, \\ Stevens Institute of Technology, Hoboken, New Jersey 07030, USA
}

\begin{abstract}
We employ a quantum Langevin equation approach to establish Bloch-type dynamical equations, on a fully microscopic basis, to investigate the measurement of the state of a coupled quantum dot qubit by a nearby quantum point contact. The ensuing Bloch equations allow us to analyze qubit relaxation and decoherence induced by measurement, and also determine the noise spectrum of the meter output current with the help of a quantum regression theorem, at arbitrary bias-voltage and temperature. Our analyses provide a clear resolution of a recent debate concerning the occurrence of a quantum oscillation peak in the noise spectrum.

PACS numbers: 73.63.Kv, 03.65.Ta, 03.65.Yz
\end{abstract}

The quantum point contact (QPC) has been proposed and designed as an efficient, practical weak continuous measurement device (meter) 1, 2, 3, 4, 4, 5, 6, 7]. Recently, theoretical analyses of the detector noise spectrum have been carried out under arbitrary bias-voltage and temperature conditions using a perturbative Green's function method [8, 9], also a quantum jump technique [10], and a Gurvitz-type master equation approach with a multisubspace ansatz 11], in which some results have led to disagreements and remain inconsistent [12]. Therefore, this problem requires further study.

Here, we revisit this issue employing a recently developed generic quantum Langevin equation approach [13], which we approximate to establish a set of Bloch-type dynamical equations describing the time evolutions of qubit variables explicitly in terms of the response and correlation functions of the detector variables. These Bloch equations provide analytical expressions for the biasvoltage and temperature $T$-dependent relaxation and decoherence of the qubit induced by measurement. We then evaluate the detector current and frequency-independent shot noise within 2nd-order perturbation theory, and they are shown to fulfill the nonequilibrium fluctuationdissipation (NFD) theorem 14. Finally, we calculate the frequency-dependent noise using a quantum regression theorem (QRT) based on the derived Bloch equations [15].

The system we examine is a double quantum dot charged qubit $c_{1}$ and $c_{2}$ coupled with a nearby lowtransparency QPC detector. In order to properly account for dissipative effects in the qubit evolution, we write the total Hamiltonian of the system in terms of the eigenstate basis of the qubit, i.e. $c_{\alpha}=\cos \frac{\vartheta}{2} c_{1}+\sin \frac{\vartheta}{2} c_{2}$, $c_{\beta}=\sin \frac{\vartheta}{2} c_{1}-\cos \frac{\vartheta}{2} c_{2}$, with $\vartheta=\tan ^{-1} \frac{\Omega}{\delta}(\Omega$ is the tunnelcoupling between two quantum dots with energies $\pm \delta$ ):

$$
\begin{aligned}
& H_{q}=\Delta \sigma_{z}, \quad H_{\mathrm{m}}=\sum_{\eta, \mathbf{k}} \varepsilon_{\eta \mathbf{k}} c_{\eta \mathbf{k}}^{\dagger} c_{\eta \mathbf{k}}, \\
& H_{\mathrm{I}}=\sum_{i=1,2} Q_{i}^{z} F_{Q^{z}}+Q_{i}^{+} F_{Q^{+}}+Q_{i}^{-} F_{Q^{-}}+Q_{i}^{\hat{1}} F_{Q^{\hat{1}}},
\end{aligned}
$$

where $\Delta=2 \sqrt{\delta^{2}+\Omega^{2}}$ measures the energy difference between two eigen-energies, i.e. the Rabi frequency; $c_{\eta \mathbf{k}}^{\dagger}$ $\left(c_{\eta \mathbf{k}}\right)$ is the electron creation (annihilation) operator in lead $\eta(=L, R)$ of the QPC with a flat density of states $\rho_{\eta} ; Q_{i}^{z}=-Q_{i}(t) \cos \vartheta, Q_{i}^{+}=Q_{i}^{-}=-\frac{1}{2} Q_{i}(t) \sin \vartheta, Q_{i}^{\hat{1}}=$ $\frac{W}{\chi} Q_{i}(t)$ with $Q_{1}(t) \equiv \chi \sum_{\mathbf{k}, \mathbf{q}} c_{L \mathbf{k}}^{\dagger} c_{R \mathbf{q}}$ and $Q_{2}(t) \equiv Q_{1}^{\dagger}(t)$ are the generalized co-ordinates; $W$ and $\chi$ are the direct and the qubit-modified tunneling matrix elements of the QPC, respectively, which are taken as constants; $F_{Q^{z}}=\hat{\sigma}_{z}=\frac{1}{2}\left(c_{\alpha}^{\dagger} c_{\alpha}-c_{\beta}^{\dagger} c_{\beta}\right), F_{Q \mp}=\hat{\sigma}_{ \pm}=c_{\alpha(\beta)}^{\dagger} c_{\beta(\alpha)}$, and $F_{Q^{\hat{1}}}=\hat{1}$ (the unit operator) are the generalized forces. Here, the terms $Q^{ \pm} F_{Q^{ \pm}}$describe energy-exchange (inelastic) processes during measurement, in which a qubit-state transition takes place from one eigenstate to the other one due to the measurement. Note that our setup, Eq. (11), is similar with those of previous studies [6, 7, 8, 10].

In our derivation of Bloch-type dynamical equations for the qubit variables, operators of the qubit and the detector are first expressed formally by integration of their Heisenberg equations of motion (EOM), exactly to all orders in the tunnel coupling and qubit-detector coupling constants, $W$ and $\chi$. Next, under the assumption that the time scale of decay processes is much slower than that of free evolutions, we replace the time-dependent operators involved in the integrals of these EOM's approximately in terms of their free evolutions (Markov approximation). Finally, these EOM's are expanded in powers of coupling constants up to second order, taking the convenient and compact form 13 : 


$$
\begin{gathered}
\left\langle\dot{\hat{\sigma}}_{z}(t)\right\rangle=-\frac{1}{2} \sin \vartheta \int_{-\infty}^{t} d \tau \sum_{c, n, l}\left\{\frac{1}{2} \theta(\tau)\left\langle\left[Q_{n o}(t), Q_{l o}^{c}\left(t^{\prime}\right)\right]_{+}\right\rangle_{m}\left\langle\left[\hat{\sigma}_{+}^{o}(t)-\hat{\sigma}_{-}^{o}(t), F_{Q^{c}}^{o}\left(t^{\prime}\right)\right]_{-}\right\rangle_{q}\right. \\
\left.+\frac{1}{2} \theta(\tau)\left\langle\left[Q_{n o}(t), Q_{l o}^{c}\left(t^{\prime}\right)\right]_{-}\right\rangle_{m}\left\langle\left[\hat{\sigma}_{+}^{o}(t)-\hat{\sigma}_{-}^{o}(t), F_{Q^{c}}^{o}\left(t^{\prime}\right)\right]_{+}\right\rangle_{q}\right\}, \\
\left\langle\dot{\hat{\sigma}}_{ \pm}(t)\right\rangle= \pm i \Delta\left\langle\hat{\sigma}_{ \pm}(t)\right\rangle \mp \int_{-\infty}^{t} d \tau \sum_{c, n, l}\left\{\frac{1}{2} \theta(\tau)\left\langle\left[Q_{n o}(t), Q_{l o}^{c}\left(t^{\prime}\right)\right]_{+}\right\rangle_{m}\left\langle\left[\cos \vartheta \hat{\sigma}_{ \pm}^{o}(t)-\sin \vartheta \hat{\sigma}_{z}^{o}(t), F_{Q^{c}}^{o}\left(t^{\prime}\right)\right]_{-}\right\rangle_{q}\right. \\
\left.+\frac{1}{2} \theta(\tau)\left\langle\left[Q_{n o}(t), Q_{l o}^{c}\left(t^{\prime}\right)\right]_{-}\right\rangle_{m}\left\langle\left[\cos \vartheta \hat{\sigma}_{ \pm}^{o}(t)-\sin \vartheta \hat{\sigma}_{z}^{o}(t), F_{Q^{c}}^{o}\left(t^{\prime}\right)\right]_{+}\right\rangle_{q}\right\},
\end{gathered}
$$

where the summation notation $c$ is over $\{z, \pm, \hat{1}\}$, and $n, l$ are over $\{1,2\}$; the statistical average $\langle\cdots\rangle_{m(q)}$ is in regard to the detector (qubit) variables; and the super(sub)script " $O$ " signifies free evolution of the corresponding variables; $\tau=t-t^{\prime}$. In conjunction with free evolution, the qubit dynamics are modified by the measurement process in a way that relates to the response function, $R_{n l}\left(t, t^{\prime}\right)$, and correlation function, $C_{n l}\left(t, t^{\prime}\right)$, of free meter variables, which are defined as:

$$
\begin{aligned}
& R_{n l}\left(t, t^{\prime}\right)=\frac{1}{2} \theta(\tau)\left\langle\left[Q_{n o}(t), Q_{l o}\left(t^{\prime}\right)\right]_{-}\right\rangle_{m}, \\
& C_{n l}\left(t, t^{\prime}\right)=\frac{1}{2} \theta(\tau)\left\langle\left[Q_{n o}(t), Q_{l o}\left(t^{\prime}\right)\right]_{+}\right\rangle_{m} .
\end{aligned}
$$

The nonvanishing correlation functions can be readily expressed in terms of reservoir Fermion distribution functions of the meter, and their Fourier transforms are 13:

$$
R_{12 / 21}(\omega)=g_{1}(\omega \pm V), \quad C_{12 / 21}(\omega)=g_{1} T \varphi\left(\frac{V \pm \omega}{T}\right)
$$

with $g_{1}=\pi \rho_{L} \rho_{R} \chi^{2} / 2$ and $\varphi(x)=x \operatorname{coth}(x / 2) . V$ is the bias-voltage applied between the left and right leads of the meter. We use units with $\hbar=k_{B}=e=1$.

For long time scale of interest, making the replacement $\int_{-\infty}^{t} d \tau \Longrightarrow \int_{-\infty}^{\infty} d \tau$, these EOM's, Eq. (2), can be further simplified as [here, we write $\left\langle\hat{\sigma}_{z( \pm)}\right\rangle \rightarrow \sigma_{z( \pm)}$ ]:

$$
\begin{aligned}
\dot{\sigma}_{z} & =-\frac{1}{T_{1}} \sigma_{z}+\Gamma_{z} \sigma_{p}+c_{z} \\
\dot{\sigma}_{ \pm} & = \pm i \Delta \sigma_{ \pm}-\frac{1}{T_{2}} \sigma_{ \pm}+\Gamma_{d} \sigma_{\mp}+\Gamma_{\|} \sigma_{z}+c_{\|}
\end{aligned}
$$

with $\sigma_{p(m)}=\sigma_{+} \pm \sigma_{-}, \Gamma_{z}=\frac{1}{4} \sin 2 \vartheta C^{+}(0), \quad \Gamma_{d}=$ $\frac{1}{2 T_{1}}, \Gamma_{\|}=\frac{1}{2} \sin 2 \vartheta C^{+}(\Delta), c_{z}=-\frac{1}{2} \sin ^{2} \vartheta R^{+}(\Delta), c_{\|}=$ $\frac{1}{4} \sin 2 \vartheta R^{+}(\Delta)\left[C(R)^{ \pm}=C(R)_{12} \pm C(R)_{21}\right]$, and the relaxation rate $\frac{1}{T_{1}}$ and decoherence rate $\frac{1}{T_{2}}$ are given by:

$$
\frac{1}{T_{1}}=\sin ^{2} \vartheta C^{+}(\Delta), \frac{1}{T_{2}}=\frac{1}{2 T_{1}}+\cos ^{2} \vartheta C^{+}(0) .
$$

It is readily seen that the relaxation time $\left(T_{1}\right)$ stems completely from inelastic measurement events, which is conceptually consistent with the physical definition of qubit relaxation. These inelastic processes also contribute to qubit decoherence with the partial rate, $1 / 2 T_{1}$. In contrast, elastic processes do not induce relaxation but do contribute to pure decoherence with the partial rate $\cos ^{2} \vartheta C^{+}(0)$. In the case of $\vartheta=0$ (no inter-dot hopping, $\Omega=0$ ), the relaxation rate is, of course, zero, meaning that the qubit is completely localized, $\frac{1}{T_{2}}=C^{+}(0)$, and $\sigma_{z}^{\infty}= \pm \frac{1}{2}$ (depending on the initial state), $\sigma_{ \pm}^{\infty}=0$; On the other hand, if $\vartheta=\pi / 2$ [the symmetric (S) case, $\delta=0], \frac{1}{T_{1}}=C^{+}(\Omega)$ and $\frac{1}{T_{2}}=\frac{1}{2 T_{1}}$. We have the general steady-state solutions of Eqs. (6a) and (6b) as:

$$
\sigma_{z}^{\infty}=-\frac{R^{+}(\Delta)}{2 C^{+}(\Delta)}, \quad \sigma_{ \pm}^{\infty}=0 .
$$

The Markov approximation employed in the derivation of Eqs. (6) requires that (1) measurement-induced decay of the qubit $\Gamma_{d} \lesssim \Delta$; and (2) rapid internal decay of the two electrodes, $1 / \tau_{c} \gtrsim V$ (coarse-graining assumption), which implies that the noise spectrum in the following calculation is meaningful only for low frequencies $\omega \lesssim V$.

The tunneling current operator through the meter is defined as the time rate of change of charge density, $N_{L}=$ $\sum_{\mathbf{k}} c_{L \mathbf{k}}^{\dagger} c_{L \mathbf{k}}$, in the left lead:

$$
\begin{aligned}
J_{L}(t)=\dot{N}_{L}= & -i\left[\left(Q_{1}^{z}-Q_{2}^{z}\right) \hat{\sigma}_{z}+\left(Q_{1}^{+}-Q_{2}^{+}\right) \hat{\sigma}_{-}\right. \\
& \left.+\left(Q_{1}^{-}-Q_{2}^{-}\right) \hat{\sigma}_{+}+\left(Q_{1}^{\hat{1}}-Q_{2}^{1}\right) \cdot \hat{1}\right] .
\end{aligned}
$$

Using linear-response theory we have

$$
\begin{aligned}
I= & \left\langle J_{L}(t)\right\rangle=-i \int_{-\infty}^{t} d t^{\prime}\left\langle\left[J_{L}(t), H_{\mathrm{I}}\left(t^{\prime}\right)\right]_{-}\right\rangle_{m, q} \\
= & 2 A_{1} R^{-}(0)+\left[\frac{1}{2} \sin ^{2} \vartheta-\frac{W}{\chi} \sin \vartheta \sigma_{p}\right] R^{-}(\Delta) \\
& +\left[\sin ^{2} \vartheta \sigma_{z}-\frac{1}{4} \sin 2 \vartheta \sigma_{p}\right] C^{-}(\Delta)
\end{aligned}
$$

with $A_{1}=\frac{1}{4} \cos ^{2} \vartheta-\frac{2 W}{\chi} \cos \vartheta \sigma_{z}-\frac{W}{2 \chi} \sin \vartheta \sigma_{p}+\left(\frac{W}{\chi}\right)^{2}$. In the case of $\vartheta=0$, the steady-state current is $I_{l(r)}=$ $V\left(g_{0}+g_{1} \pm 2 \sqrt{g_{0} g_{1}}\right)$ with $g_{0}=2 \pi \rho_{L} \rho_{R} W^{2}$, where $I_{l(r)}$ 
means the QPC current when dot 2(1) of the qubit is occupied by electron; For the $\mathrm{S}$ case $\delta=0$, we have

$$
I=g_{0} V+g_{1} V\left(1-\frac{\Delta}{V} \frac{C^{-}(\Delta)}{C^{+}(\Delta)}\right) .
$$

To address the noise spectrum of a "symmetric" detector, we employ its definition as the Fourier transform of the current-current correlation function, $S(\tau)$, which can be calculated using linear-response theory,

$$
S(\omega)=\int_{-\infty}^{\infty} d \tau e^{i \omega \tau} \frac{1}{2}\left\langle\left[\delta J_{L}(t), \delta J_{L}\left(t^{\prime}\right)\right]_{+}\right\rangle_{m, q},
$$

with $\delta J_{L}(t)=J_{L}(t)-\left\langle J_{L}(t)\right\rangle$. It is well-known that the shot noise spectrum consists of the $\omega$-independent Schottky noise $S_{0}$ (the pedestal) and the $\omega$-dependent part. Substituting the current operator of Eq. (9) into Eq. (12) and using the definitions Eqs. (3)-(5), we obtain the $\omega$-independent noise, $S_{0}$, as:

$$
\begin{aligned}
S_{0}= & 2 A_{1}^{\infty} C^{+}(0)+\left[\frac{1}{2} \sin ^{2} \vartheta-\frac{W}{\chi} \sin \vartheta \sigma_{p}^{\infty}\right] C^{+}(\Delta) \\
& +\left[\sin ^{2} \vartheta \sigma_{z}^{\infty}-\frac{1}{4} \sin 2 \vartheta \sigma_{p}^{\infty}\right] R^{+}(\Delta),
\end{aligned}
$$

where the first terms on the right hand side of Eq. (10) at $t \rightarrow \infty$ and Eq. (13) obey the NFD relation, while the other two terms represent the generalized NFD relation due to energy-exchange processes involved in the course of measurement [14].

From Eq. (10), it is clear that the time evolution of the qubit variables determines the temporal behavior of the meter current, and is consequently responsible for the frequency-relevant part of the noise spectrum. To calculate the $\omega$-dependent noise, it is thus necessary to evaluate the two-time correlation functions $\sigma_{a b}(\tau)=\left\langle\sigma_{a}(t+\tau) \sigma_{b}(t)\right\rangle(a, b=z, p, m)$ using the QRT starting with the dynamic equations (6). The QRT states that given closed-form equations of motion of one-time averages of a set of operators $\mathcal{O}_{j}$ :

$$
\frac{d}{d \tau}\left\langle\mathcal{O}_{j}(t+\tau)\right\rangle=\sum_{k} L_{j, k}\left\langle\mathcal{O}_{k}(t+\tau)\right\rangle+\lambda_{j},
$$

then the two-time averages of $\mathcal{O}_{j}$ with any other operator, $\mathcal{P}$, also obey the same equations [15]:

$$
\frac{d}{d \tau}\left\langle\mathcal{O}_{j}(t+\tau) \mathcal{P}(t)\right\rangle=\sum_{k} L_{j, k}\left\langle\mathcal{O}_{k}(t+\tau) \mathcal{P}(t)\right\rangle+\lambda_{j}\langle\mathcal{P}(t)\rangle
$$

Because the solutions of interest here involve long time behavior after the initial turn-on, we replace the initial values of $\sigma_{a b}(0)=\left\langle\sigma_{a}(t) \sigma_{b}(t)\right\rangle$ and the last term $\langle\mathcal{P}(t)\rangle$ in Eq. (15) with their respective steady-state values. Once these equations for $\sigma_{a b}(\tau)$ are solved and substituted into the definition, Eq. (12), we calculate the frequencydependent noise spectrum $S(\omega)$.
There is no general analytic expression for the noise spectrum, except in two special cases: In the absence of coupling, $\Omega=0, I(t)=\left[\frac{1}{2}+2\left(\frac{W}{\chi}\right)^{2}\right] R^{-}(0)-\frac{4 W}{\chi} \sigma_{z}(t)$, but $\dot{\sigma}_{z}=0$, therefore the total noise is independent of $\omega$ with $S=I_{l(r)} \operatorname{coth}(V / 2 T)$; On the contrary, for the S system $\delta=0$, we have $I(t)=I_{0}+I_{1}(t)=\left(g_{0}+g_{1}\right) V-A \sigma_{p}(t)+$ $B \sigma_{z}(t)$, with $A=\frac{4 W}{\chi} g_{1} V$ and $B=C^{-}(\Delta)$. Moreover, since $\sigma_{z}(t)$ is decoupled from $\sigma_{ \pm}(t)$ in Eqs. (6) due to $\Gamma_{z}=0$ and $\Gamma_{\|}=0$, we have $\sigma_{p z}(\tau)=\sigma_{z p}(\tau)=0$, and then $S(\tau)=\operatorname{Re}\left[A^{2} \sigma_{p p}(\tau)+B^{2} \sigma_{z z}(\tau)\right]-\left\langle I_{1}\right\rangle^{2}$. Finally, the total noise spectrum, $S(\omega)=S_{0}+S_{1}(\omega)+S_{2}(\omega)$, is

$$
\begin{gathered}
S_{0}=g_{0} V \operatorname{coth}\left(\frac{V}{2 T}\right)+\frac{1}{2} C^{+}(\Delta)\left[1-\left(\frac{2 g_{1} \Delta}{C^{+}(\Delta)}\right)^{2}\right], \\
S_{1}(\omega)=\frac{(\delta I)^{2} \Gamma_{d} \Delta^{2}}{\left(\omega^{2}-\Delta^{2}\right)^{2}+4 \Gamma_{d}^{2} \omega^{2}}, \\
S_{2}(\omega)=\left[1-\left(\frac{2 g_{1} \Delta}{C^{+}(\Delta)}\right)^{2}\right] \frac{\left[C^{-}(\Delta)\right]^{2} \Gamma_{d}}{\omega^{2}+4 \Gamma_{d}^{2}},
\end{gathered}
$$

with $\delta I=4 V \sqrt{g_{0} g_{1}}$. The pedestal shot noise, $S_{0}$, is identical with the previously found result $[\underline{8}]$. For the frequency-relevant noise spectrum in a $\mathrm{S}$ qubit, the decoupled qubit dynamics give rise to two distinct components: $S_{1}(\omega)$ stems from the transverse qubit dynamics, $\sigma_{p}$, i.e. elastic and inelastic measurement-induced qubit decoherence, which is similar to the calculation of Ref. $\underline{6}$ but augmented with our specific predictions for $V$ - and $T$-dependent parameters, $\Gamma_{d}$ and $\delta I$; while $S_{2}(\omega)$ is generated by the longitudinal qubit dynamics $\sigma_{z}$ (qubit relaxation), which is exclusively due to inelastic processes in measurement. Clearly, our $S_{1}(\omega)$ is similar to Shnirman's Eq. (35) [8] except for the reduction factor. Moreover, our $S_{2}(\omega)$ coincides with $C_{3}(\omega)$ [Eq. (39)] [] under the same limit conditions, and also vanishes for $V<\Delta$ at $T=0$. In our model, $S_{2}(\omega)$ is at least one order of magnitude smaller than $S_{1}(\omega)$, irrespective of $V, T$, and $g_{1} / g_{0}$ [inset in Fig. 1(a)], indicating that the detector spectrum mainly reflects $\mathrm{S}$ qubit decoherence behavior. Noticing that our different low-bias behavior of $S_{1}(\omega)$ as compared to Ref. 8 is due to the coarse-graining assumption, which requires $V \gtrsim \Delta$ to guarantee accurate noise spectrum at $\omega \sim \Delta$.

Figure 1(a) plots measurement-induced qubit decoherence effects on the noise spectrum in the maximal meterresponse case $g_{1}=g_{0}=0.25$. It may be observed that with increasing $\Gamma_{d}$ (corresponding voltages $V / \Delta=1.5$, 2.0, 3.0, and 4.0), $S(\omega)$ changes from a Lorentzian-type function, with a peak located at the qubit Rabi frequency $\omega=\Delta$, to the spectrum shape centered at $\omega=0$, indicating the Zeno effect regime. This behavior can be understood as follows: (1) a low voltage applied to QPC can not provide sufficient dissipation (small $\Gamma_{d}$ ) to suppress the Rabi oscillation of a $\mathrm{S}$ qubit, thus a peak occurs in 

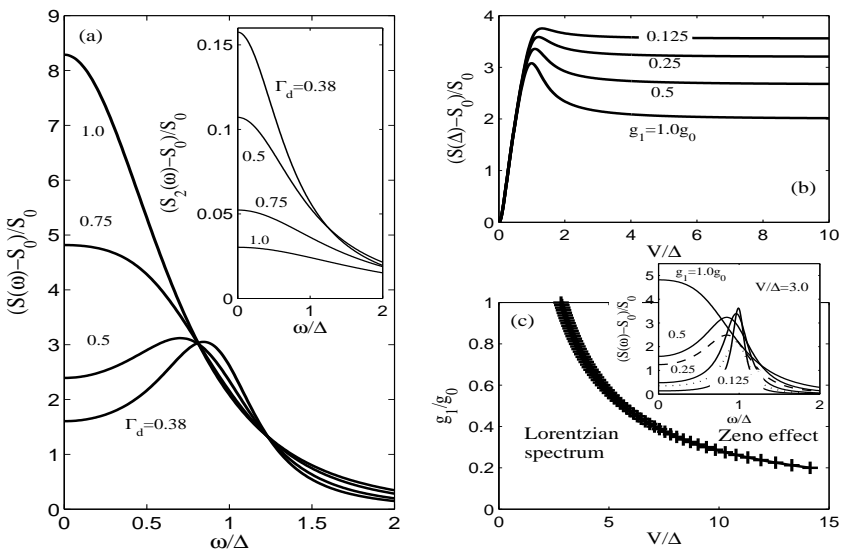

FIG. 1: (a) $S(\omega)$ as function of frequency $\omega / \Delta$ for various $\Gamma_{d}$ values. The parameters we use in calculation are: $g_{0}=g_{1}=$ 0.25 and $T=0.1 \Delta$; (b) The peak-to-pedestal ratio vs. biasvoltage; (c) Ranges of various functional forms for the noise spectrum for a given QPC transparency $g_{0}=0.25$. Inset: $S(\omega)$ vs. $\omega / \Delta$. Solid lines denote results for various $g_{1} / g_{0}$ values at $g_{0}=0.25$. The dashed and dotted curves are for $g_{1}=g_{0}$ but lower values of $g_{0}=0.125$ and 0.06 , respectively.

the noise spectrum contrary to the previous prediction of Ref. 8 as mentioned above; on the other hand, (2) a high voltage can freeze the qubit due to enhanced relaxation, indicating appearance of the Zeno effect. The specific value of voltage dividing the two regimes naturally depends on the QPC-qubit coupling for a given QPC transparency $g_{0}$. Based on Eq. 16b), the transition occurs under the condition $\Gamma_{d}>\Delta / \sqrt{2}$, which leads to a rough transition boundary involving $\chi / W$ and $V$ $\left(\log \frac{g_{1}}{g_{0}} \propto-\log V\right)$ as shown in Fig. 1(c) for the QPC with a fixed transparency $g_{0}=0.25$. The Lorentzian spectrum regime extends to a wider range of $V$ for systems with weaker detector-qubit coupling $\chi$. The inset of Fig. 1(c) exhibits the fact that the spectrum changes from the Zeno regime back to the Lorentzian regime as $g_{1}$ decreases. We should notice that the transition boundary depends on $g_{0}$ due to $g_{1} \leq g_{0}$, though $\Gamma_{d}$ is independent of $g_{0}$. In the high-voltage limit, $V \gg \Delta$, $T$, we can still obtain the Lorenzian noise spectrum of detector output via applying a QPC with very low conductance, $g_{0} \ll \Delta / V$, to guarantee $\Gamma_{d} \ll \Delta$. This remark is shown by dashed and dotted lines in the inset of Fig. 1(c).

Another interesting feature is the quantum upper bound of 4 for signal-to-noise ratio (SNR) in the qubit measurement, which was first pointed out by Korotkov and Averin in the high voltage limit $[\underline{6}$. From Fig. 1(b), we observe that (1) the SNR increases rapidly initially with rising voltage, and reaches a maximum value less than 4 (depending on $g_{1}$ ) around $V=\Delta ;(2)$ increasing qubit-QPC coupling $g_{1}$ results in reduction of the SNR due to enhancement of $S_{0}$, and the SNR becomes 2 in the high voltage limit in the case of maximal response $g_{1}=g_{0}$. These results indicate that low QPC trans- parency $g_{0}$, weak meter-qubit coupling, $g_{1}$, and $V \geq \Delta$ are necessary for an efficient meter.

The efficiency of a meter also depends decisively on temperature [6]. The spectrum peak at $\omega=\Delta$ in Fig. 1(a) will gradually disappear with increasing $T$ (not shown here), which is ascribed to enhanced detector-induced decay, $\Gamma_{d}$. From Eq. (7), $\Gamma_{d}$ is independent of $V$ at $T<\Delta / 2$ and $V<\Delta$, implying that the QPC is always a good meter for a $\mathrm{S}$ qubit at low temperature and bias-voltage.
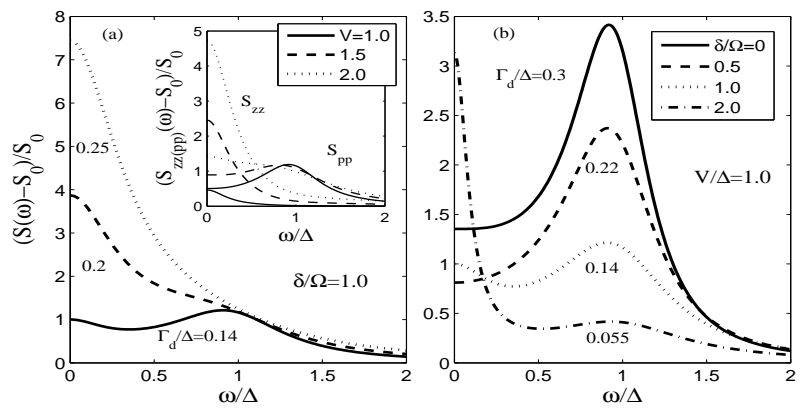

FIG. 2: (a) $S(\omega)$ vs. $\omega / \Delta$ for an asymmetric qubit with $\delta=\Omega$ at various bias-voltages; (b) $S(\omega)$ for different qubits at $V=1.0 \Delta$. Other parameters: $g_{0}=g_{1}=0.25, T=0.1 \Delta$.

The noise spectra for asymmetric (A) qubits are summarized in Fig. 2. In contrast to the $\mathrm{S}$ qubit, the A qubit longitudinal dynamics start to play a dominant role in the spectrum around $\omega=0$ when $V \geq \Delta\left[S_{z z}\right.$ in the inset of Fig. 2(a)], making $S(\omega)$ approach a zero-frequency maximum more quickly, albeit that the transverse spectrum, $S_{p p}$, still has a peak at $\omega=\Delta$. This is to say that breakdown of the resonance condition causes the A qubit measurement to enter into the Zeno effect regime more quickly, even with $\Gamma_{d} \ll \Delta$.

In conclusion, we have analyzed the noise output spectrum of a QPC-qubit measurement system for arbitrary voltage and temperature conditions using a general quantum-operator Langevin equation approach to derive Bloch equations for the system. In contrast to the results in Ref. 10, which improperly ignores quantum interference between elastic and inelastic processes of measurement, our calculations indicate that qubit oscillations always cause a peak in the QPC noise spectrum at the Rabi frequency, $\Delta$, for a $S$ qubit, because the qubit coherence dynamics dominate contributions to the noise spectrum (if the condition $\Gamma_{d}<\Delta / \sqrt{2}$ is satisfied), which agrees with previous studies in the high voltage limit $[$ ] . However, the coherent peak at the Rabi frequency is suppressed by the relaxation dynamics of an A qubit even when $\Gamma_{d} \ll \Delta$. Our analyses provide physical insight into qualitative criteria for design of an efficient detector: (1) low transparency $g_{0}$ of a QPC; $(2)$ weak meter-qubit coupling, $g_{1}$; (3) small asymmetry ratio, $\delta / \Omega$; (4) relatively low temperature.

This work was supported by Projects of the National Science Foundation of China and the Shanghai Municipal 
Commission of Science and Technology. NJMH was supported by the DURINT Program administered by the US Army Research Office, DAAD Grant No.19-01-1-0592.

[1] E. Buks, R. Schuster, M. Heiblum, D. Mahalu, and V. Umansky, Nature 391, 871 (1998); D. Sprinzak, E. Buks, M. Heiblum, and H. Shtrikman, Phys. Rev. Lett. 84, 5820 (2000); M.A. Kalish, M. Heiblum, A. Silva, D. Mahalu, and V. Umansky, Phys. Rev. Lett. 92, 156801 (2004).

[2] R.J. Schoelkopf, P. Wahlgren, A.A. Kozhevnikov, P. Delsing, and D.E. Prober, Science 280, 1238 (1998).

[3] Y. Nakamura, Yu.A. Pashkin, and J.S. Tsai, Nature 398, 786 (1999).

[4] D. Mozyrsky and I. Martin, Phys. Rev. Lett. 89, 18301 (2002).

[5] S. Pilgram and M. Büttiker, Phys. Rev. Lett. 89, 200401 (2002); A.N. Jordan, M. Büttiker, cond-mat/0505044

[6] A.N. Korotkov, Phys. Rev. B 63, 85312 (2001); A.N. Korotkov and D.V. Averin, Phys. Rev. B 64, 165310 (2001);
R. Ruskov and A.N. Korotkov, Phys. Rev. B 67, 75303 (2003).

[7] H.S. Goan, G.J. Milburn, H.M. Wiseman, and H.B. Sun, Phys. Rev. B 63, 125326 (2001); H.S. Goan and G.J. Milburn, Phys. Rev. B 64, 235307 (2001).

[8] A. Shnirman, D. Mozyrsky, and I. Martin, cond-mat/0211618 Europhys. Lett. 67, 840 (2004).

[9] L.N. Bulaevskii, M. Hruška, and G. Ortiz, Phys. Rev. B 68, 125415 (2003).

[10] T.M. Stace and S.D. Barrett, Phys. Rev. Lett. 92, 136802 (2004); cond-mat/0309610

[11] X.Q. Li, P. Cui, and Y.J. Yan, Phys. Rev. Lett. 94, 66803 (2005).

[12] D.V. Averin and A.N. Korotkov, cond-mat/0404549 T.M. Stace and S.D. Barrett, cond-mat/0406751

[13] B. Dong, N.J.M. Horing, and H.L. Cui, Phys. Rev. B 72, 165326 (2005).

[14] D. Rogovin and D.J. Scalapino, Ann. Phys. (N.Y.) 86, 1 (1974); E.V. Sukhorukov, G. Burkard, and D. Loss, Phys. Rev. B 63, 125315 (2001).

[15] M.O. Scully and M.S. Zubairy, Quantum optics, (Cambridge University Press, Cambridge, 1997). 